\title{
Snoring as main symptoms of peripheral primitive neuroectodermal tumor in oropharynx:a case report with review of literature
}

\section{Xin-xiang Ma}

Shaoxing Keqiao Women's and Children's Hospital

Chuan-xin Zhang ( $D$ zcx1560@163.com)

Shaoxing Keqiao Women's and Children's Hospital https://orcid.org/0000-0003-1575-5265

Yi-fan Ren

Shaoxing Keqiao Women's and Children's Hospital

Yu-lin Zhang

Shaoxing Keqiao Women's and Children's Hospital

Ji-ai Zheng

Shaoxing Keqiao Women's and Children's Hospital

\section{Case report}

Keywords: case report, snoring, peripheral primitive neuroectodermal tumor, oropharynx, children

Posted Date: August 30th, 2021

DOl: https://doi.org/10.21203/rs.3.rs-847261/v1

License: (c) (i) This work is licensed under a Creative Commons Attribution 4.0 International License. Read Full License 


\section{Abstract}

Introduction

In children, snoring is often associated with hypertrophied tonsils and adenoids. There was a case in a girl who did not suffer from hypertrophy adenoids, but from a peripheral primitive neuroectodermal tumor (pPNET) in the oropharynx. pPNET often occurs in the deep soft tissues of the trunk, paravertebral, lower limbs and retroperitoneum. It is rarely located in children's oropharynx and makes snoring.

\section{Patient concerns}

We report a case of a 4-years-old girl who complained of slurred speech and snoring. Her serum showed normal results, computed tomography (CT) and magnetic resonance imaging (MRI) revealed a mass lesion in oropharynx. By using histology and immunohistochemistry, cluster of differentiation 99 (CD99), vimentin, friend leukemia integration 1 (FLI-1), integrase interactor-1 and a-smooth muscle actin were positive, but epithelial membrane antigen, erythroblast transformation-specific (ETS) related gene (ERG), NK homeobox 2.2, Wilms' tumor 1 and S100-protein were negative. And the rearrangement of the Ewing sarcoma breakpoint region 1 (EWSR1) gene was proved in the test of fluorescent.

Diagnosis

She was diagnosed as a peripheral primitive neuroectodermal tumor.

Interventions and outcome

The girl had been treated with surgery and chemotherapy. She has been well for 24 months without any signs of disease.

Conclusion

In children who snore, we should not only think of hypertrophied adenoids, but also the possibility of pPNETs in oropharynx. CT scan or MRI should be conducted in time, as well as histology and immunohistochemistry to avoid misdiagnosis.

\section{Introduction}

Snoring results from turbulent airflow and vibration of soft tissues. In children, the primary cause of snoring is hypertrophied tonsils and/or adenoids. ${ }^{[1]}$ There was a child who developed slurred speech and snoring. She was diagnosed as "sinusitis and hypertrophy adenoids " by pediatrician. Antibiotics and montelukast were given to her for some time, but did not help. After a computed tomography (CT) and magnetic resonance imaging (MRI) examination were performed in our hospital, a large mass was found in her oropharynx. And pathological examination revealed that the tumor was extraosseous Ewing's sarcoma (EES). This is a rare case of EES in children's oropharynx. Herein, we report it as follows. 


\section{Case Presentation}

A 4-years-old girl visited the hospital with the chief complaint of slurred speech for more than 1 year and snoring for 6 months. One year ago, the child's speech was not clear, the parents took her to see doctor in clinic. The doctor thought it might be related to speech retardation, and advised her to receive language training. Half a year later, the child developed snoring, accompanied by nasal congestion and purulent discharge. She was diagnosed as "acute sinusitis and adenoid hypertrophy" by pediatrician in another hospital. Here, she was treated with amoxicillin and clavulanate potassium $60 \mathrm{mg} / \mathrm{kg} / \mathrm{d}$, q8h, po, and nasal irrigation with normal saline, bid, for 2 weeks, but the effect was not obvious. So, the antibiotic was replaced with azithromycin, $5 \mathrm{mg} / \mathrm{kg} / \mathrm{d}, \mathrm{d} 1-3$, qd, po. The first oral dose is doubled. Every 7 days is a course of treatment, a total of 3 courses. The child's nasal congestion, nasal discharge disappeared, but slurred speech and snoring remained. In the following months, the girl was also treated with montelukast, her snoring did not abate but grew louder, and she developed a breath-holding and restless sleep. Thus, her parents became very anxious and took her to our hospital.

She had shortness of breath,but no fever, cough, or headache. A large pink mass was seen in her oropharynx, and other physical examination did not reveal any significant. A routine blood and other laboratory tests showed no abnormalities. We suspected an oropharyngeal capillary hemangioma, so we performed a CT scan of her neck. CT scan showed that a low and heterogeneous density mass located in the oropharynx, its CT value was $48 \mathrm{Hu}$, and its size was $31 \times 36 \times 22 \mathrm{~mm}$. The oropharyngeal space was markedly narrowed (Fig. 1A). MRI images (Fig. 1B, C) demonstrated isointense signals on T1-weighted imaging, and hyperintense signals on T2-weighted imaging. The right boundary of the lesion was unclear, the left protruded toward the larynx, and the adjacent pharyngeal cavity was obviously narrow. The lesion was obviously enhancement after enhanced scan. No lesions were found in other soft tissues or in bone tissue. All suggested oropharyngeal tumor. Then, the tumor was partly resected by surgery, it was brittle and hard, and there was necrosis within it. Histology showed the malignant tumor composed of small, round, blue cells with vesicular nuclei and scanty neoplasm, and the cells were heteromorphosis (Fig. 1D). Immunohistochemically, the tumor cells were positive for cluster of differentiation 99 (CD99), cytokeratin, cyclinD1, vimentin, friend leukemia integration 1 (FLI-1), integrase interactor-1, ki67, CD31, CD34 and asmooth muscle actin, while epithelial membrane antigen, erythroblast transformation-specific (ETS) related gene (ERG), NK homeobox 2.2, Calponin, CD20, synaptophysin, chromogranin-A, Wilms' tumor 1 and S100-protein were negative. Cells released from paraffin block were tested by using the Ewing's sarcoma break-apart probe. It showed a rearrangement of Ewing sarcoma breakpoint region 1 (EWSR1). All findings supported a diagnosis of extraosseous Ewing's sarcoma (EES)/pPNET. The patient underwent systemic therapy. The chemotherapy regimen was vincristine-pirarubicin-cyclophosphamide (CAV) and ifosfamide-etoposide (IE). The CAV/IE regimen calls for circles of vincristine $\left(1.5 \mathrm{mg} / \mathrm{m}^{2}, \mathrm{~d} 1\right)$, cyclophosphamide $\left(1.0 \mathrm{~g} / \mathrm{m}^{2}, \mathrm{~d} 1\right)$ with mesna rescue, and pirarubicin $\left(50 \mathrm{mg} / \mathrm{m}^{2}, \mathrm{~d} 1\right)$ alternating with circles of ifosfamide ( $3 \mathrm{~g} / \mathrm{m}^{2}$, plus mesna, d1-3) and etoposide $\left(150 \mathrm{mg} / \mathrm{m}^{2}, \mathrm{~d} 1-3\right)$, given every 21 days for three cycles with evaluation of response. Surgical resection was performed to remove the tumor as completely as possible after images showed it had shrunk, and followed by further chemotherapy (CAV 
regimen for another six cycles). During the period of chemotherapy, she had occasional nausea and vomiting, which could be relieved by the treatment of granisetron, and the other major adverse reaction was bone marrow suppression, which recovered after symptomatic treatment. Currently, receiving regular follow-up care, she speaks clearly, sleeps without snoring. She is well with no signs of disease for 24 months.

\section{Discussion}

In 1921, James Ewing ${ }^{[2]}$ firstly reported Ewing's sarcoma (ES), and in 1969 Tefft $^{[3]}$ reported extraosseousEwing's sarcoma(EES)/peripheral primitive neuroectodermal tumors (pPNETs). EES is a malignant tumor derived from neuroectodermal that is clinically diagnosed as a PPNET,and considered a special clinical manifestation of ES. EES/pPNETs are small, blue, round cells from neural crest cells, and all of them belong to the Ewing's sarcoma family. pPNET often occurs in the deep soft tissues of the trunk, paravertebral, lower limbs and retroperitoneum, and can be also found in the kidney, pancreas, ovaries and testes. ${ }^{[4,5]}$ It is rare in the head and neck, especially in the larynx.

There is no characteristic clinical presentation of pPNETs, the main manifestations are only related to tumor location, size, and invasiveness. The images and serum biomarkers are also nonspecific. On CT images, the tumor's density was uniform or heterogeneous, and the size of tumors depends on where they are located. The boundaries between the lesion and adjacent tissues are unclear. Maximum intensity projection image reconstruction can show tortuous blood vessels in tumors on enhanced CT images. ${ }^{[6]}$ On MRI images, most tumors have isointense to slightly hyperintense T1-weighted signal and heterogeneous hyperintense T2-weighted signal, ${ }_{1}^{[7]}$ and have obvious enhancement when enhanced scan. On fluid-attenuated inversion recovery images, the lesion mixed high signal intensity. ${ }^{[7,8]}$ However, intratumoral calcification was rare in pPNETs. ${ }^{[9]}$

Because of unspecific clinical manifestations, the diagnosis of pPNETs mainly depends on histopathology. Under the light microscope, small blue round cells in tumor are visible, they are tightly arranged in cords or nests to form rosettes and pseudorosettes (Homer-Wright rosettes). In cytogenetics, almost all of pPNETs are defined by translocation of chromosome 22, a fusion of the Ewing's sarcoma breakpoint protein (EWS/EWSR1), located at 22q12, and a gene of the erythroblast transformationspecific (ETS) family of transcription factors. In approximately $90 \%$ of pPNETs, the fusion gene is EWSFLI1, which formed by a chromosomal translocation $t(11 ; 22)$ (q24; q12). FLI1, located at 11q24, is one of the members of the ETS family. In approximately $10 \%$ of pPNETs, the fusion gene is EWS-ERG, by translocation $t(21 ; 22)$ (q22; q12), which results in fusion of the EWS gene on chromosome 22 to the ERG gene on chromosome 21. Here, the ERG gene substitutes for FLI1. ${ }^{[10]}$ In addition, there are a small number of ETS family genes, such as ETV1 (7p22) and E1AF (17q12), which form fusion genes with EWS. ${ }^{[11]}$ Immunohistochemically, ES/pPNETs strongly express the membranous immunostaining of CD99 that is sensitive but not specific. CD99 is almost always present in these tumors. As reported, the positivity of CD99 is in up to $92 \%{ }^{[12]} \mathrm{FLI}-1$, one of the ETS family transcription factors, is highly expressed in 
ES/pPNETs. It has been reported that the protein expression in over $70 \%$ of ES/PNET with a specificity of over $90 \%$. Therefore, it suggests that antibodies to FLI-1 may play a very valuable role in the diagnosis of ES/PNET. ${ }^{[13,14]}$ Due to the high sensitivity of CD99 and the high specificity of FLI-1, Sarah Chiang et al. ${ }^{[15]}$ considered the tumor, showed both membranous CD99 and nuclear FLI-1 expression, was more likely to harbor an EWSR1 rearrangement, and this is consistent with ES/pPNETs. Currently, an important basis on the diagnosis of ES/pPNETs is the presence of at least two neuromarkers in the Homer-Wright chrysanthemum structure or/and immunohistochemistry. ${ }^{[16]}$

pPNETs are usually treated with a combination of surgery, radiotherapy and chemotherapy. Surgery should completely remove the tumor as much as possible. Chemotherapy may use the CAV-IE regimen with vincristine-pirarubicin-cyclophosphamide (CAV) and ifosfamide-etoposide (IE) alternately. Radiotherapy should be based on the age of the child, the size and location of the tumor, the response to chemotherapy, the type of histology and residual lesions. The radiation dose usually ranged from 30 to 66 Gy.

pPNETs deteriorate rapidly and have poor prognosis. Jurgens et al. ${ }^{[17]}$ reported a 3-year disease-free survival rate of $56 \%( \pm 11 \%)$. Patients with pPNET often developed distant metastases and local recurrence within 2-3 years after surgery. It is well known the pPNETs of the parapharyngeal space are extremely rare. $\mathrm{Lu}^{[18]}$ reported a similar case of cervical ganglioneuroblastoma of the parapharyngeal space with progressive inspiratory dyspnea and dysphagia in a 4-year-old female,the girl was treated with surgery and remained healthy for more than one year. Another similar case of an 8-year-old girl was reported by Divya Khosla. ${ }^{[19]}$ Although after chemotherapy and radiotherapy treatment, but she eventually died of lung and brain metastasis due to the deterioration of the disease.

Because ES/pPNETs have non-specific clinical manifestations, it is easy to cause misdiagnosis if pediatricians lack an understanding of them. The case we reported was misdiagnosed by pediatricians in many hospitals as speech retardation, sinusitis and adenoid hypertrophy, due to slurred speech and sleep snoring. When the diagnosis was made, the tumor had been getting huge. This lesson cannot be forgotten. Therefore, for a child who is slurred speech and snoring, we should not only think of hypertrophied tonsils and adenoids, but also tumors in oropharynx. It is necessary to give CT or MRI examination on head, neck or chest, so as not to take a misdiagnosis of the disease.

\section{Conclusion}

In children who snore, we should not only think of hypertrophied adenoids, but also the possibility of pPNETs compressing surrounding tissue in oropharynx. pPNETs have high malignancy, aggressivity, and poor prognosis. Their clinical manifestations are non-specific, and the diagnosis depends on tissue biopsy. Hence, CT scan or MRI should be conducted in time, as well as cytogenetic and molecular biological detection. pPNETs cases are treated with multi-agent treatment, including surgery, chemotherapy and/or radiation therapy. 


\section{Abbreviations}

pPNET = peripheral primitive neuroectodermal tumor, ES $=$ Ewing's sarcoma, EES $=$ extraosseous Ewing's sarcoma, $\mathrm{CT}$ = computed tomography, $\mathrm{MRI}$ = magnetic resonance imaging, FLI-1= Friend leukemia integration 1, EWS= Ewing's sarcoma breakpoint protein, EWSR1=Ewing sarcoma breakpoint region $1, E T S=$ erythroblast transformation-specific, ERG= ETS-related gene, $C D=$ cluster of differentiation .

\section{Declarations}

\section{Ethics approval and consent to participate}

The ethics committee of Shaoxing Keqiao Women's and Children's Hospital has approved the study.

\section{Consent for publication}

The girl's parent consents for publication.

\section{Availability of data and materials}

Not applicable.

\section{Competing interests}

The authors have no competing interests.

\section{Funding}

There is no funding.

\section{Author's contributions}

Chuan-xin Zhang conceived the topic idea, wrote introduction, discussion and conducted literature review. Xin-xiang Ma identified and managed the case, conducted literature review and contributed to writing the case presentation and discussion. Yi-fan Ren contributed to the revision of the article. Yu-lin Zhang and Ji-ai Zheng contributed to writing part of the case presentation and provided figures.

\section{Acknowledgements}

Not applicable.

\section{References}

1. Gursanscky J, Boston M, Kamani T. A snoring child. BJM. 2017 May 18; 375: j2124. doi:10.1136/bmj. j2124. 
2. Ewing J. The Classic: Diffuse endothelioma of bone. Proceedings of the New York Pathological Society. 1921: 12: 17. Clin Orthop Relat Res.2006,450:25 - 7.

3. Crist WM, Raney RB, Tefft M, et al. Soft tissue sarcomas arising in the retroperitoneal space in children. A report from the Intergroup Rhabdomyosarcoma Study (IRS) Committee. Cancer. 1985;56(8):2125-32.

4. Karpate A, Menon S, Basak R, Yuvaraja TB, Tongaonkar HB, Desai SB. Ewing sarcoma/primitive neuroectodermal tumor of the kidney: clinicopathologic analysis of 34 cases. Ann Diagn Pathol,2012,16(4):267-74.

5. Gupta P, Dhingra KK, Singhal S, Khurana N, Mandal S, Mohta A. Primary primitive neuroecto dermal tumour (PNET) of the testis: an unsuspected diagnosis. Pathology. 2010;42(2):179-81.

6. Liu J, Zhao YL, Song SQ, Li ZH, Li PL. Primitive neuroectodermal tumors: a clinical and radiological analysis of six cases. Quant Imaging Med Surg. 2019;9(4):722-9.

7. Gupta P, Hari S, Thulkar S. Imaging spectrum of peripheral primitive neuroectodermal tumours. Singapore Med J. 2013;54(8):463-9.

8. Chu WC,ReznikovB, Lee EY, Grant RM, Cheng FWT, Babyn P. Primitive neuroectodermal tumour (PNET) of the kidney: a rare renal tumour in adolescents with seemingly characteristic radiological features. PediatrRadiol. 2008;38(10):1089-94.

9. Xu Qian Xu, Kai L, Shaodong C, Gaohong M. Hong, Li Jingjing. Radiological and clinicopathological features of pPNET. Eur J Radiol. 2013;82:e888-93.

10. Lessnick SL, Ladanyi M. Molecular pathogenesis of Ewing sarcoma: new therapeutic and transcriptional targets. Annu Rev Pathol. 2012;7:145-59.

11. Sandberg AA. Bridge JA.Updates on cytogenetics and molecular genetics of bone and soft tissue tumors: Ewing sarcoma and peripheral primitive neuroectodermal tumors. Cancer Genet Cytogenet. 2000;123(1):I-26.

12. Louati S, Senhaji N, Chbani L, Bennis S. EWSR1 Rearrangement and CD99 Expression as Diagnostic Biomarkers for Ewing/PNET Sarcomas in a Moroccan Population. Dis Markers 2018, Sep18;2018:7971019. doi: 10.1155/2018/7971019. eCollection 2018.

13. Folpe AL, Hill CE, Parham DM,O'Shea PA, Weiss SW. Immunohistochemical detection of FLI-1 protein expression: a study of 132 round cell tumors with emphasis on CD99-positive mimics of Ewing's sarcoma/primitive neuroectodermal tumor. Am J Surg Pathol. 2000;24(12):1657-62.

14. Mhawech-Fauceglia P. Herrumnn F,Bshara W,et a1.Friend leukaemia integration-1 expression in malignant and benign tumours: a multiple tumour tissue microarray analysis using polyclonal antibody. J Clin Pathol. 2007;60(6):694-700.

15. Chiang S, Snuderl M, Kojiro-Sanada, et al. Primitive Neuroectodermal Tumors of the Female Genital Tract: A Morphologic, Immunohistochemical and Molecular Study of 19 Cases. Am J Surg Pathol. 2017;41(6):761-72.

16. Schmidt D, Herrmann C, Jürgens $H$, Harms D. Malignant peripheral neuroectodermal tumor and its necessary distinction from Ewing's sarcoma. A report from the Kiel Pediatric Tumor Registry. Cancer. 
1991;68(10):2251-9.

17. Jurgens $H$, Bier $V$, Harms D, et al. Malignant peripheral neuroectodermal tumors. A retrospective analysis of 42 patients. Cancer. 1988;61:349-57.

18. Lu D, Liu J, Chen Y, Chen F, Yang H. Primary cervical ganglioneuroblastoma: A case report. Medicine. 2018;97(12):e0090. doi:10.1097/ MD.0000000000010090.

19. Khosla D, Verma S, Punia RS, et al. Extraosseous Ewing's Sarcoma of the Parapharyngeal Space - A Rare Entity - with Review of Literature. Iran J Otorhinolaryngol. 2019;31(102):51-4.

\section{Figures}



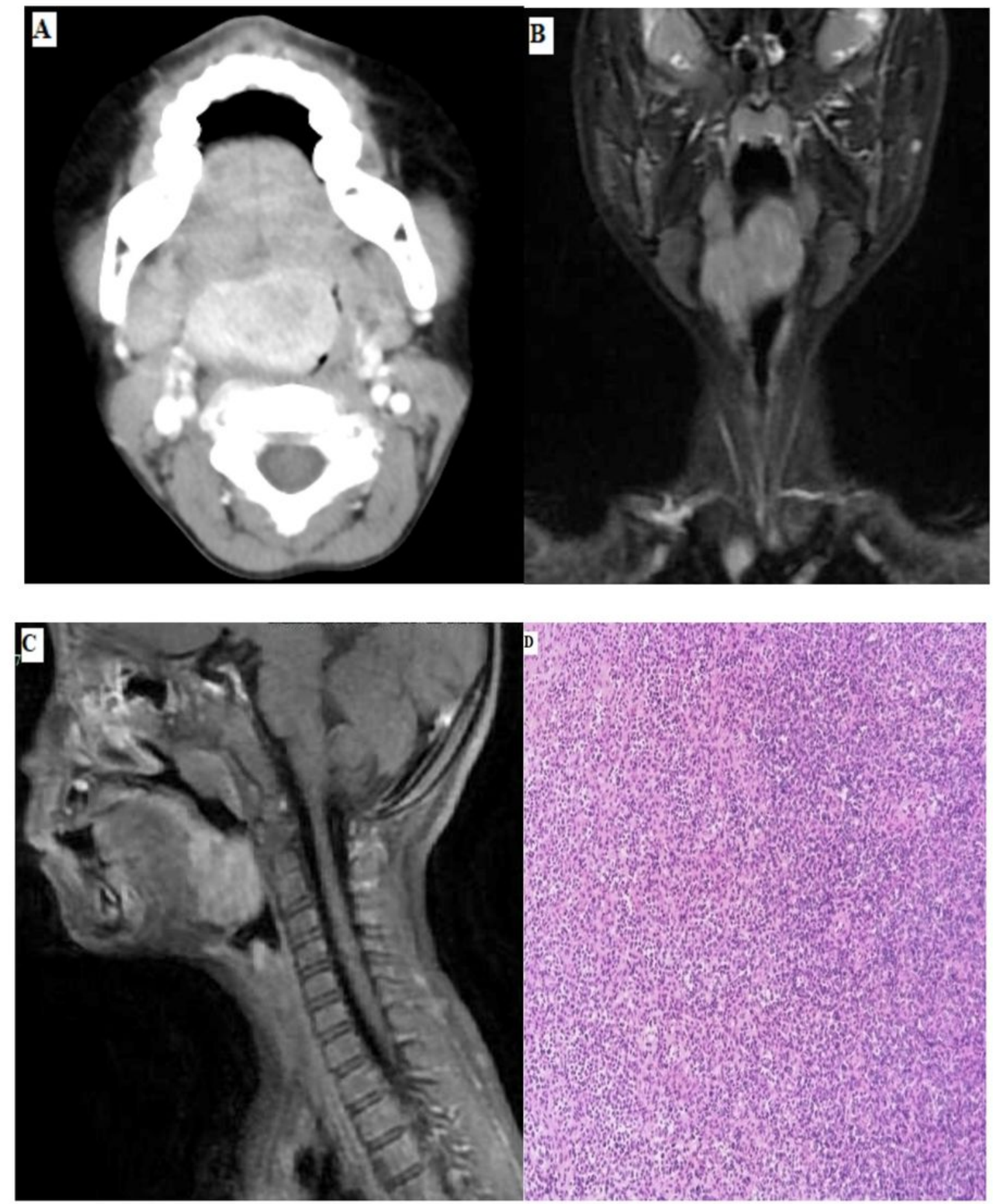

Figure 1

(A) CT imaging showed a low-density mass locates in the larynx, and the density is heterogeneous. The mass measured $31 \times 36 \times 22 \mathrm{~mm}$ in size, and the oropharyngeal and laryngeal pharyngeal spaces were markedly narrowed. (B, C) MR images demonstrate hyperintense signals on T2-weighted imaging. The lesion is marked enhancement after enhanced scan. (D) The small-, round-, blue-cell tumor, can be 
demonstrated biochemically, ultrastructurally, and by immunoperoxidase techniques (Hematoxylineosin stain, $\times 400)$. 